\title{
Obtención de recubrimientos autolubricados por proyección térmica de plasma atmosférico (APS) como alternativa a los lubricantes líquidos
}

\author{
J.M. GUILEMANY, J. FERNÁNDEZ, J. NAVARRO, J.M. MIGUEL \\ CPT, Centro de Proyección Térmica. Ingeniería de Materiales. Dept. Enginyeria Química i Metal.lurgia. \\ Universitat de Barcelona. C/ Martí i Franquès, 1. E-08028 Barcelona.
}

\begin{abstract}
La obtención de recubrimientos con bajos coeficientes de fricción, es una innovadora respuesta a la creciente demanda industrial de alternativas para los tradicionales sistemas lubricados con aceites. Para este fin, la introducción de un sólido lubricante, en una matriz con buenas propiedades mecánicas, ha de conferir al conjunto un bajo coeficiente de fricción y mejorar la resistencia al desgaste del recubrimiento, favoreciendo además, la utilización a elevadas temperaturas, en las que los lubricantes líquidos convencionales presentan limitaciones. En el Centro de Proyección Térmica (CPT) de la Universidad de Barcelona se han obtenido mediante proyección de Plasma Atmosférico (APS) recubrimientos autolubricados de Ni-Grafito y $\mathrm{Cr}_{2} \mathrm{O}_{3}-\mathrm{CaF}_{2}$, estudiando con especial interés las propiedades y el efecto que produce el grafito y el $\mathrm{CaF}_{2}$ en las matrices metálica y cerámica respectivamente. Se ha determinado en los dos casos que el desgaste originado en los recubrimientos es bajo, si bien los coeficientes de fricción varían ostensiblemente en función del sistema indicado. Los resultados obtenidos indican que el recubrimiento de Ni-Grafito posee un comportamiento idóneo capaz de competir con los tradicionales lubricantes líquidos.
\end{abstract}

Palabras clave: Autolubricados, Tribología, Ni-Grafito, $\mathrm{Cr}_{2} \mathrm{O}_{3}-\mathrm{CaF}_{2}$, Proyección Térmica.

Self-lubricated coatings obtained by Atmospheric Plasma Spraying (APS), as a liquid lubricants alternative

The low friction coefficient coatings are a new approach to substitution of oil lubricated systems. The introduction of a solid lubricant in a matrix with good mechanical properties gives to the system a low friction coefficient and improves the wear resistance of the coating. These new systems can be used at high temperatures where liquid lubricants fail.

$\mathrm{Ni}$-Graphite and $\mathrm{Cr}_{2} \mathrm{O}_{3}-\mathrm{CaF}_{2}$ self-lubricated coatings have been produced by Atmospheric Plasma Spray (APS), in the Thermal Spray Center of the University of Barcelona. The effect of graphite and $\mathrm{CaF}_{2}$ on the metallic matrix and coatings properties has been studied. It was observed that the wear was low in both systems, but the friction coefficients are very different.

The results show that the Ni-Graphite coatings has an good behaviour and can be used as alternative to liquids lubricants.

Keywords: Self-lubricated, Tribology, Ni-Graphite, $\mathrm{Cr}_{2} \mathrm{O}_{3}-\mathrm{CaF}_{2}$. Thermal Spray.

\section{INTRODUCCIÓN}

Las técnicas de proyección térmica permiten modificar mediante la generación de recubrimientos, las propiedades tribológicas de piezas, cuyas superficies debe ser sometida a condiciones de trabajo muy específicas y en algunos casos extremas. En esta técnica se trasmite energía térmica y cinética a un material en forma de polvo, hilo o varilla, que es fundido y proyectado hasta el substrato (previamente granallado), donde llega en estado pastoso o semipastoso. La contracción de las partículas durante la solidificación da lugar a un anclaje de tipo mecánico con el substrato (1).

Los recubrimientos autolubricados obtenidos por proyección térmica están compuestos por una fase lubricante sólida finamente dispersa dentro de una matriz metálica o cerámica que posea buenas propiedades mecánicas y buenas propiedades de resistencia al desgaste (2)(3). El lubricante sólido confiere al conjunto un bajo coeficiente de fricción, una mejor resistencia al desgaste y una disminución del daño producido en las piezas que trabajan a fricción. Estas mejoras introducidas por el lubricante sólido dentro del sistema, son fundamentales para minimizar la energía perdida en sistemas que trabajan a fricción, para aumentar la vida de trabajo de piezas disminuyendo total o parcialmente la cantidad de lubricante líquido y por tanto minimizar el impacto ambiental y económico que genera el uso y posterior tratamiento de lubricantes líquidos.

Actualmente los lubricantes sólidos mas utilizados son el $\mathrm{MoS}_{2}, \mathrm{WS}_{2}, \mathrm{CaF}_{2}$ y el Grafito. Además, el uso de este tipo de lubricantes, permite trabajar a elevadas temperaturas, en las que los lubricantes líquidos tradicionales se degradarían (4).

En el presente trabajo se han estudiados recubrimientos de Ni-Grafito (5) y de $\mathrm{Cr}_{2} \mathrm{O}_{3}$ con diferentes cantidades de $\mathrm{CaF}_{2}$ (6)

\section{MÉTODO EXPERIMENTAL}

Como material de aporte se han utilizado polvos comerciales de $75 \% \mathrm{Ni}-25 \%$ Grafito (en peso), referencia comercial Metco 307NS y $\mathrm{Cr}_{2} \mathrm{O}_{3^{\prime}}$, referencia comercial FST C650-32, con la cual se realizaron diferentes mezclas mecánicas 
con $\mathrm{CaF}_{2}\left(5 \%, 10 \%, 15 \%\right.$ y $25 \%$ en peso de $\left.\mathrm{CaF}_{2}\right)$. Para la obtención de los recubrimientos se utilizó un equipo APS Plasma-Technik A-3000S con una antorcha F4.

La sección transversal de los recubrimientos se ha preparado mediante embutición de las diferentes probetas en resina conductora y posterior corte, desbaste y pulido, y se ha estudiado mediante microscopía electrónica de scanning (JEOL JSM-5310 trabajando a 20kV) y microscopía óptica (Olympus BH2-UMA).

Las medidas de dureza (sección transversal) han sido realizadas con un microdurómetro Matzusawa MXT-OX dotado de un indentador Vickers, con cargas de $300 \mathrm{~g}$, aplicadas durante $15 \mathrm{~s}$.

Los ensayos de resistencia al desgaste por fricción, han sido realizados con un equipo Ball-on-disk siguiendo la norma ASTM G99-90. Se utilizó una bola de acero martensítico con una dureza de 585 Vickers y $11 \mathrm{~mm}$ de diámetro. Los ensayos de desgaste se realizaron en una cámara cerrada a temperatura y humedad controlada $\left(20^{\circ} \mathrm{C}\right.$ y HR menor del $\left.20 \%\right)$. La carga aplicada es de $15 \mathrm{~N}$ y el diámetro de giro es de $16 \mathrm{~mm}$, con una velocidad de giro de $131 \mathrm{rpm}$, lo que representa una velocidad lineal de $0.11 \mathrm{~m} / \mathrm{s}$. La distancia de ensayo es de $1000 \mathrm{~m}$ y los coeficientes de fricción han sido calculados con respecto a los 200 últimos metros.

Los caminos de desgaste fueron evaluados mediante microscopía electrónica de barrido (SEM), y las medidas sobre pérdida de volumen, anchura y profundidad de huella de desgaste producidos en el ensayo de resistencia al desgaste por fricción se han determinado mediante Interferometría de luz blanca de barrido (SWLI), utilizando un equipo Zygo New View100.

\section{RESULTADOS Y DISCUSIÓN}

Para la obtención de los recubrimientos se ha utilizado como gases plasmágenos mezclas de $\mathrm{H}_{2}$ y Ar. En las figuras I y II puede observarse la sección transversal de los recubrimientos de Ni-grafito y de $\mathrm{Cr}_{2} \mathrm{O}_{3}-5 \% \mathrm{CaF}_{2}$ (en peso). Se aprecia en ambos casos una estructura compacta, con un nivel de porosidad bajo, comprendido entre el $2 \%$ y el $4 \%$ (Tabla 1), indicando que en los dos casos, las condiciones de proyección son correctas. Se puede observar que la porosidad

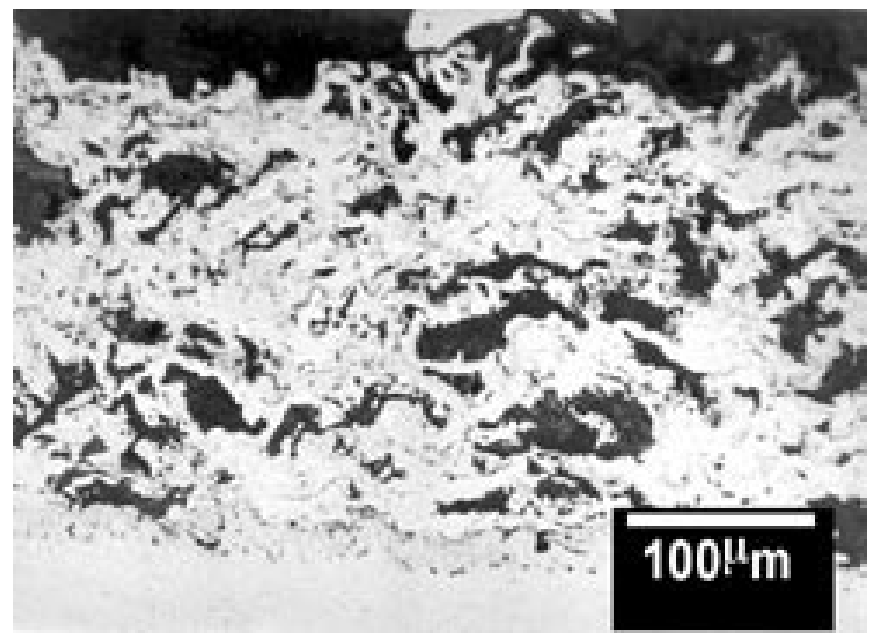

Fig. 1.- Vista general del recubrimiento de Ni-Grafito.

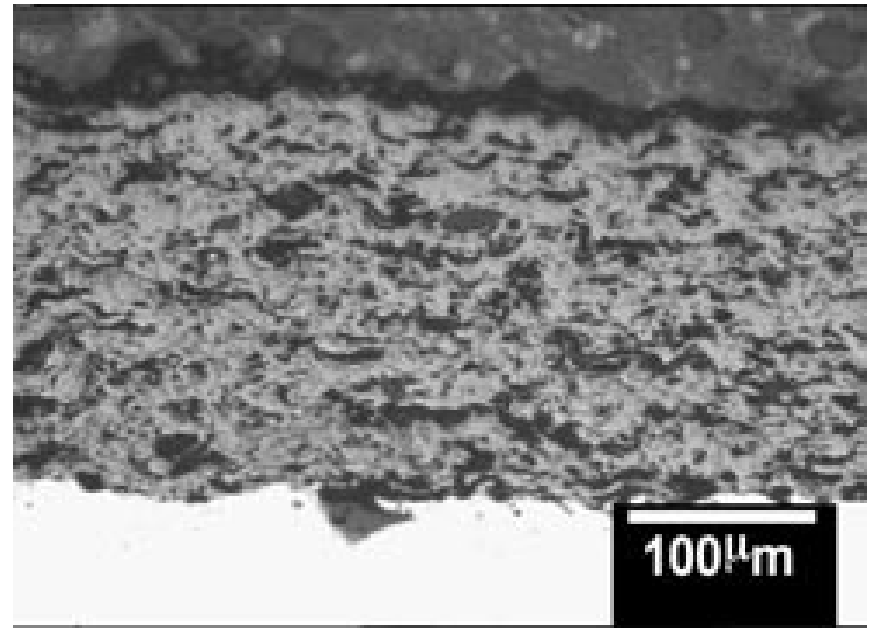

Fig.2.- Vista general del recubrimiento de $\mathrm{Cr}_{2} \mathrm{O}_{3}-5 \% \mathrm{CaF}_{2}$.

TABLA I. PROPIEDADES DE LOS RECUBRIMIENTOS.

\begin{tabular}{|l|l|l|l|}
\hline & $\mathrm{HVN}_{300}$ & Desviación Standard & $\%$ Porosidad \\
\hline Ni-Grafito & 44 & 31 & 4.0 \\
\hline $\mathrm{Cr}_{2} \mathrm{O}_{3}$ & 845 & 21 & 2.0 \\
\hline $\mathrm{Cr}_{2} \mathrm{O}_{3}-5 \% \mathrm{CaF}_{2}$ & 820 & 74 & 2.3 \\
\hline $\mathrm{Cr}_{2} \mathrm{O}_{3}-10 \% \mathrm{CaF}_{2}$ & 655 & 88 & 2.4 \\
$\mathrm{Cr}_{2} \mathrm{O}_{3}-15 \% \mathrm{CaF}_{2}$ & 570 & 25 & 2.8 \\
$\mathrm{Cr}_{2} \mathrm{O}_{3}-25 \% \mathrm{CaF}_{2}$ & 435 & 21 & 2.8 \\
\hline
\end{tabular}

está localizada preferentemente en la fase lubricante, debido fundamentalmente a arranques producidos en la preparación de las probetas para la observación óptica (desbaste y pulido). En el caso del Ni-grafito se puede apreciar además una acumulación de porosidad en la interfacie grafito-metal, debido a la mala adaptabilidad del grafito a la estructura en el momento de ser depositado.

La estructura es homogénea en los dos casos, observándose una correcta dispersión de la fase lubricante dentro de la matriz metálica (Ni) y cerámica (cromia), si bien se puede apreciar que las partículas de grafito son de mayor tamaño que las de $\mathrm{CaF}_{2}$, debido a la naturaleza del polvo original empleado. La interfacie no presenta poros o discontinuidades producidas por tensiones residuales de la capa.

Los valores de microdureza aparecen expresados en la Tabla I. Se aprecia en el caso del Ni-Grafito que los valores obtenidos se sitúan por debajo de los 100 Vickers, debido fundamentalmente a la baja dureza de la fase no metálica. El valor de la desviación standard es muy elevado, debido a las elevadas dimensiones de los splats. Al realizar el ensayo de microdureza sobre éstos splats, se realizan microindentaciones sobre uno de los dos componentes del recubrimiento (sobre el grafito o sobre el Niquel), obteniéndose valores de dureza muy diferentes. Para reducir este efecto se procedió a aumentar la carga a $500 \mathrm{~g}$, pero los valores obtenidos eran muy bajos, debido a que parte de la carga se disipaba por la creación de roturas entre las partículas depositadas, resultando una medida de dureza inferior a la real.

En el caso de los recubrimientos de Cromia, se puede observar como al aumentar el contenido de fase lubricante, disminuye la dureza global del recubrimiento de tal forma

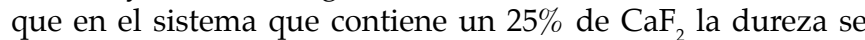
reduce a la mitad de la que presenta el recubrimiento formado por Cromia. 
Las propiedades lubricantes de los recubrimientos se han evaluado mediante el ensayo de Ball-on-Disk (BoD). En la Figura III, se representa el valor de los coeficientes de fricción vs distancia de fricción para algunos de los recubrimientos ensayados (debido al solapamiento de las curvas de desgaste en el caso de los diferentes sistemas de $\mathrm{Cr}_{2} \mathrm{O}_{3}-\mathrm{CaF}_{2}$, sólo se ha representado la curva de $\mathrm{Cr}_{2} \mathrm{O}_{3}$ y la de la mezcla al $15 \%$ de $\left.\mathrm{CaF}_{2}\right)$. Los coeficientes de fricción obtenidos para los recubrimientos de matriz cerámica se sitúan entre 0.7-0.5, muy elevados comparados con el valor obtenido en el recubrimiento de Ni-Grafito $(\mu=0.24)$, indicando un menor efecto lubricante por parte del $\mathrm{CaF}_{2}$.

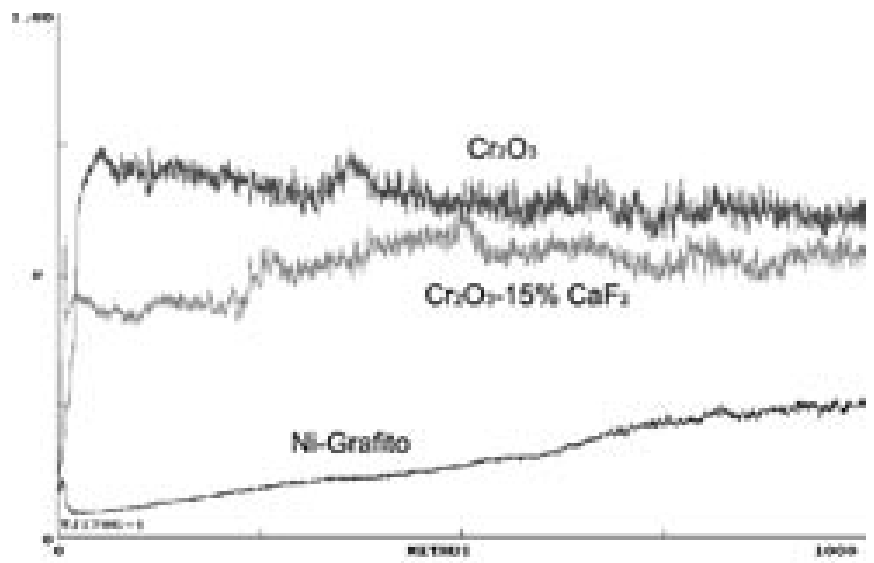

Fig.3.- Evolución de los coeficientes de fricción vs. la distancia de deslizamiento.

El estudio de los caminos de desgaste se realizó mediante Microscopía Electrónica de Barrido (SEM), para caracterizar los mecanismos responsables del deterioro de los recubrimientos. La superficie del camino de desgaste en el caso del Ni-Grafito no aparece excesivamente rallada, indicando que el mecanismo de desgaste por abrasión es moderado (Figura IV).

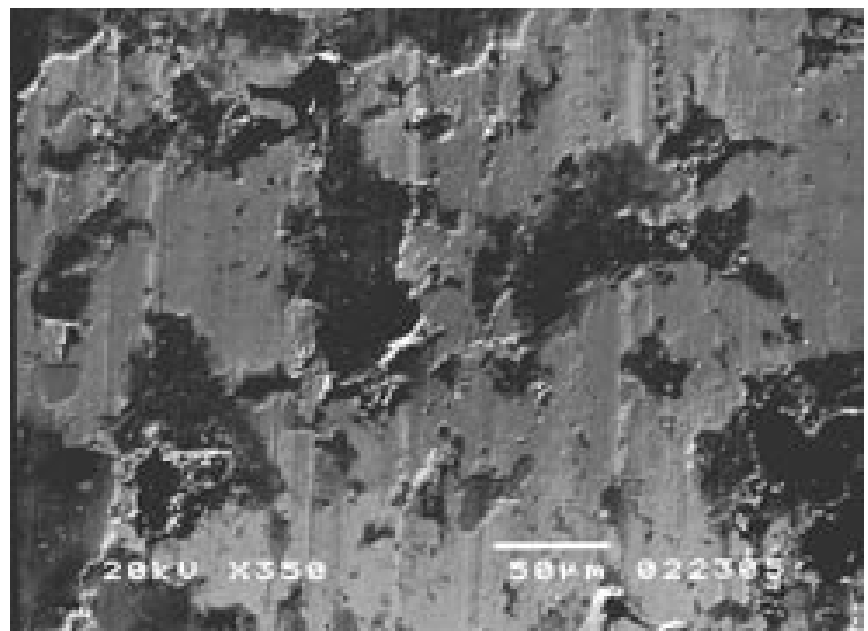

Fig.4.- Camino de desgaste. Mecanismo de desgaste abrasivo moderado

Existen zonas a lo largo del camino de tonalidad oscura, que indica que se ha depositado grafito sobre el camino, inhibiendo los mecanismos de adhesión entre el recubrimiento y la contraprobeta y minimizando de esta forma el desgaste adhesivo. Estudios mediante EDS, no revelaron la existencia de hierro a lo largo del camino (proveniente de la contraprobeta de acero), resultado que constata lo anteriormente expuesto.
En cambio, para los sistemas formados de matriz cerámica, se observa el efecto contrario. A simple vista se observa, un coloración rojiza en todo el camino de desgaste, típica de formación de óxido de hierro, que se puede comprobar mediante el estudio en SEM (Figura V, material depositado) y análisis de EDS. Este efecto se observo en todos los sistemas de matriz cerámica utilizados, indicando que existe una lubricación limitada, ya que el mecanismo de desgaste adhesivo, es el que tiene mayor importancia en este tipo de recubrimiento.

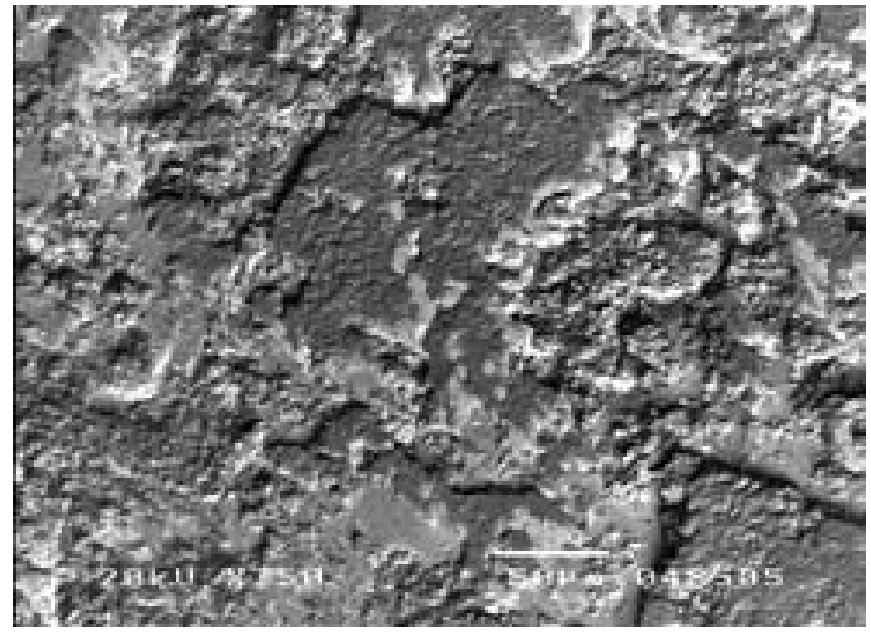

Fig.5.- Material depositado en el camino de desgaste procedente de la bola de acero

Los caminos de desgaste obtenidos en BoD fueron evaluados mediante la técnica de interferometría de luz blanca. Los parámetros de desgaste (Tabla II), sólo se pudieron obtener para el recubrimiento de Ni-Grafito (Figura VI), ya que en el caso de los recubrimientos de $\mathrm{Cr}_{2} \mathrm{O}_{3}-\mathrm{CaF}_{2}$ donde, como ya se ha dicho anteriormente, el mecanismo principal de desgaste es el adhesivo, sólo se pudo observar el material depositado sobre la superficie del recubrimiento (Figura VII).

TABLA II. PARÁMETROS DE DESGASTE. AREA DE CONTACTO DE LA BOLA DE ACERO, VOLUMEN PERDIDO DE RECUBRIMIENTO, ANCHURA Y PROFUNDIDAD DEL CAMINO DE DESGASTE

\begin{tabular}{|c|c|c|c|c|}
\hline & $\begin{array}{c}\text { Área de } \\
\text { contacto } \\
(\mathrm{mm})\end{array}$ & $\begin{array}{c}\text { Vol.Rec. } \\
\text { Perdido } \\
\left(\mathrm{mm}^{3}\right)\end{array}$ & $\begin{array}{c}\text { Anchura } \\
(\mathrm{mm})\end{array}$ & Profundidad $(\mu \mathrm{m})$ \\
\hline $\mathrm{Ni}-\mathrm{C}$ & 0.7 & 0.24 & 0.67 & 10.2 \\
\hline $\begin{array}{c}\text { Acero } \\
\text { (Substrato) }\end{array}$ & 2.0 & 3.2 & 2.0 & 52 \\
\hline
\end{tabular}

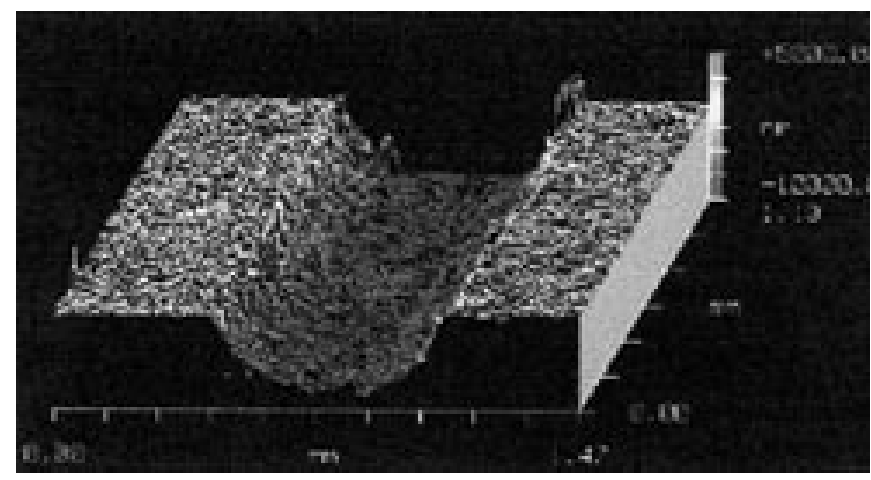

Fig.6.- Camino de desgaste de Ni-Grafito 


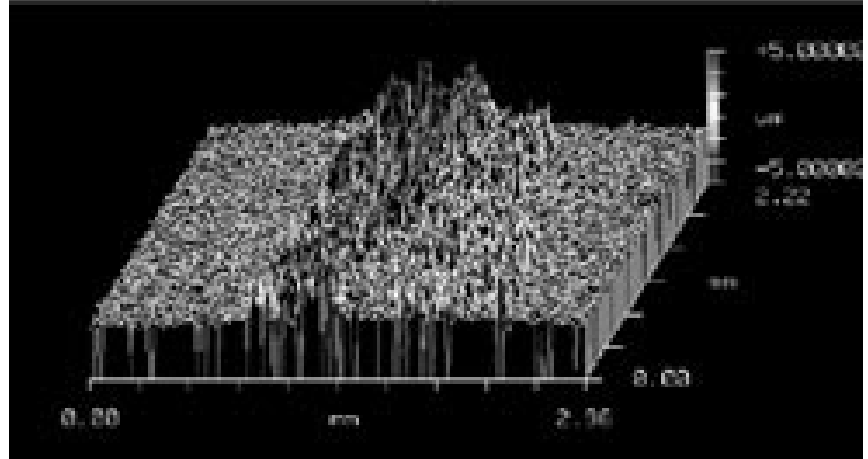

Fig.7.- Camino de desgaste de $\mathrm{Cr}_{2} \mathrm{O}_{3}-\mathrm{CaF}_{2}$.

El área de contacto de las contraprobetas de acero se midió mediante microscopía óptica a fin de evaluar el daño producido en las contraprobetas. Es destacable que el daño producido por el recubrimiento de Ni-Grafito sobre la contraprobeta de acero es muy pequeño, siendo apenas apreciable mediante microscopía óptica. Este efecto es muy importante, ya que al producir un daño tan pequeño, se amplia la expectativa de vida del conjunto. En cambio en los recubrimientos de $\mathrm{Cr}_{2} \mathrm{O}_{3} \mathrm{y}$ $\mathrm{Cr}_{2} \mathrm{O}_{3}-\mathrm{CaF}_{2}$, no ocurre lo mismo ya que los pares friccionantes quedan seriamente dañados, mostrando unas áreas de contacto muy elevadas, signo evidente de menor lubricación.

\section{CONCLUSIONES}

La utilización de recubrimientos de Ni-Grafito, minimiza el daño producido sobre la contraprobeta de acero, debido a la ausencia de mecanismos de desgaste adhesivo y abrasivo sobre la superficie de la contraprobeta (bola de acero). Se ha comprobado que el mecanismo de desgaste adhesivo se encuentra inhabilitado tanto en la contraprobeta como sobre la superficie del recubrimiento, sobre la cual se deposita una película lubricante impidiendo la microsoldadura. La bola tampoco ha sido dañada por un mecanismo abrasivo, ya que la baja dureza del recubrimiento de Ni-Grafito lo impide.

Los sistemas con matriz de $\mathrm{Cr}_{2} \mathrm{O}_{3}$ y dispersión de $\mathrm{CaF}_{2}$ presentan coeficientes de fricción por encima de 0.5 , indicando que el mecanismo de lubricación es limitado. El estudio de los caminos de desgaste mediante SEM y SWLI reveló la existencia de restos de acero proveniente de las contraprobetas, indicando que el mecanismo de desgaste principal en este tipo de recubrimientos es el desgaste adhesivo. De acuerdo con este dato se estudiaron las contraprobetas de acero, observándose una elevada pérdida de volumen.

EL Ni-Grafito ofrece las mejores características como recubrimiento autolubricado, debidoa la excelente combinación de resistencia al desgaste y bajo coeficiente de fricción.

\section{AGRADECIMIENTOS}

Los autores quieren agradecer al Ministerio de Ciencia y Tecnología la concesión de los proyectos MAT 2001-3399 y MAT 2001-3498. A la Generalitat de Catalunya el proyecto 2001SGR 00145.

\section{BIBLIOGRAFÍA}

1. L.Pawlowski. “Coating build-up” pp. 108-163 en The Science and Engineering of Thermal Spray Coatings. John Wiley\&Sons, West Sussex (Inglaterra), 1995

2. G.H. Liu, F. Robbevalloire, R. Gras, J. Blouet. "Improvement in tribological properties of chromium oxide coating at high temperature by solid lubricants". Wear, 160. 181-189. (1993)

3. S. Midorikawa. Thermal Spray Current Status and Future Trends. Vol. 1, Kobe. (1995)

4. A. Borisova, Y.Barisov, A.Tunik, L.Adeeva, E.Lugscheider, C.Herbst. "Thermal Spraying of coating containing solid lubricants" United Themal Spray Conference. 174-181 Dusseldorf (1999)

5. J.M. Guilemany, J.Navarro, C. Lorenzana, J.M. Miguel. Proceedings of the $18^{\text {th }}$ International Thermal Spray Conference, (2001) 1115-1118.

6. M.Van Stappen, M.Kerkhofs, L.M.Stals, C. Quaeyhaegens. "State of art for the industrial use of ceramic PVD coatings" Surface and coatings tecnology 75 (1-3), 629-633. (1995)

Recibido: 01.02 .03

Aceptado: 15.02.05

(Presentado en el Congreso de Materiales. Madrid, 2002) 